\title{
The Usefulness of the Pretreatment Neutrophil/ Lymphocyte Ratio as a Predictor of the 5-Year Survival in Stage 1-3 Triple Negative Breast Cancer Patients
}

\author{
Basem Azab ${ }^{a}$ Julia R. Amundson ${ }^{b}$ Alessia Ciocic Heather Stuart ${ }^{d}$ \\ Danny Yakoub ${ }^{\mathrm{e}}$ Eli Avisar ${ }^{c}$ Fredrick Moffat $^{c} \quad$ Alan S. Livingstone $^{c}$ \\ Dido Franceschic

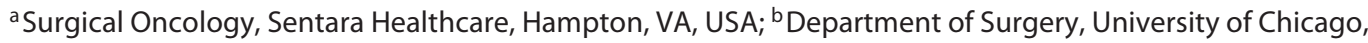 \\ Chicago, IL, USA; ' Department of Surgical Oncology, University of Miami, Miami, FL, USA; ${ }^{\text {'D }}$ epartment of \\ Surgical Oncology, University of British Colombia, Vancouver, BC, Canada; e Department of Surgical Oncology, \\ University of Tennessee, Memphis, TN, USA
}

\section{Keywords}

Neutrophil/lymphocyte ratio - Triple negative breast cancer $\cdot$ Mortality

\begin{abstract}
Background: We have previously shown that the neutrophil/lymphocyte ratio (NLR) is a predictor of survival among breast cancer patients. The aim of this study was to determine the predictive value of NLR among different nodal and chemotherapy subgroups of triple negative breast cancer (TNBC). Methods: Patients with stage 1-3 TNBC who underwent treatment from 2007 to 2014 and had blood counts prior to treatments were included. Patients were categorized into high $(\geq 2)$ and low $(<2)$ NLR groups. Primary outcomes were overall survival (OS) and disease-free survival (DFS). Results: The average follow-up time was 54 months. The high NLR group had worse OS (HR 2.8, Cl 1.3-5.9, $p<$ $0.001)$ and DFS (HR 2.3, Cl 1.2-4.2, $p<0.001)$ than the low NLR group. After adjusting for confounding variables, high NLR was an independent prognostic factor for both OS (HR 5.5, $\mathrm{Cl}$ 2.2-13.7, $p<0.0001$ ) and DFS (HR 5.2, Cl 2.3-11.6, $p<$ 0.0001 ). Categorization of TNBC patients by NLR (high vs. low) and nodal status (positive vs. negative) resulted in four groups with significantly different OS and DFS (log rank $p<$ $0.0001)$. Significant improvements in OS $(p<0.001)$ and DFS
\end{abstract}

$(p<0.001)$ were observed for patients who received chemotherapy and had high NLR but not for patients with low NLR ( $p=0.65$ and $p=0.07$, respectively). Conclusion: High pretreatment NLR is an independent predictor of poor OS and DFS among TNBC patients. Combining NLR and $\mathrm{pN}$ provides better risk stratification for TNBC patients. Chemotherapy appears to be beneficial only in patients with high NLR. Larger prospective studies are needed to validate these findings.

(c) 2020 S. Karger AG, Basel

\section{Introduction}

Over many decades, breast cancer remains a major target for research by most of the health organizations. Despite the actual declining of breast cancer mortality over the last two decades, the 2017 estimates of new cases and breast cancer-related deaths are expected to be 252,710 and 40,610, respectively [1]. The aforementioned improvement of survival among breast cancer patients was partially attributed to the current understanding of the breast molecular subtypes including the targeted treatment modalities (i.e., hormonal and HER-2-targeted therapies). However, further research is needed for the estrogen, progesterone, and HER-2 receptor-negative breast cancer patients known as the triple negative breast 
cancer (TNBC) group. TNBC comprises about 15 to $20 \%$ of the total breast cancer population [2] and is associated with poor disease-free survival (DFS) and overall survival (OS) [3]. Research efforts continued to categorize the TNBC group into seven molecular subtypes [4] and further to study the treatment response among these different subtypes $[5,6]$.

As the inflammation plays a pivot role and an integral component in the process of cancer progression and metastasis [7-9], elevated inflammatory surrogates (e.g., Creactive protein, serum amyloid $\mathrm{A}$, and serum interleukin-6) were found to correlate with reduced survival among breast cancer patients [10-12]. We previously reported a simple inflammatory marker, the elevated peripheral neutrophil/lymphocyte ratio (NLR), as an independent predictor of poor short- and long-term survival in breast cancer patients $[13,14]$. Recently, several metanalyses have demonstrated the negative impact of elevated NLR on breast cancer patients' outcomes [15-17]. Similarly, among TNBC, elevated NLR was associated with poor survival among 90 TNBC patients [18]. Moreover, Asano et al. [19] found elevated NLR as a predictor of low rate of pathological complete remission among 61 TNBC patients. While the axillary lymph node status remains a valuable prognostic factor for breast cancer patients, the aim of this study was to determine the predictive value of NLR among different pathological nodal $(\mathrm{pN})$ and chemotherapy subgroups of TNBC.

\section{Methods}

This is a retrospective longitudinal cohort study comprising of 125 consecutive women with TNBC who were undergoing treatment at the University of Miami, Jackson Memorial Hospital, and Sylvester Comprehensive Cancer Center from January 2007 to December 2014. Inclusion criteria were patients diagnosed with TNBC with data on complete blood count including leukocyte differential available before surgery and/or chemotherapy. Exclusion criteria were patients without blood count data prior to chemotherapy, presence of active infection during the pretreatment period, presence of coexisting hematological malignancies or other hematological disorders, autoimmune disorders, and patients on recent steroid therapy. Patients were followed up until July 2016, with an average follow-up time of 54 months (range 24-110).

Differential leukocyte counts were obtained by the Coulter counter ${ }^{\circledR}$ technique (Coulter Gen.S Hematology Analyzer; Beckman Coulter Corp., Hialeah, FL, USA). Data collection was accomplished by electronic chart review of each patient to obtain information on demographic characteristics, tumor characteristics, including grade and stage as well as laboratory parameters. Data on the primary endpoint (mortality) were obtained from the cancer registry and social security death index. Positive smoking status meant active or ex-smoker. The AJCC classification was obtained depending upon the tumor $(\mathrm{T})$, node $(\mathrm{N})$, and metastases $(\mathrm{M})$ status of each cancer. Complete pathological response after neoadjuvant chemotherapy was defined as absence of a residual invasive component in breast or lymph node (ypT0/is ypN0) [20]. The estrogen receptor and progesterone receptors were considered posi-
Table 1. Demographic, clinicopathological, and treatment variables according to the pretreatment NLR among TNBC patients

\begin{tabular}{|c|c|c|c|}
\hline & $\begin{array}{l}\text { Low NLR } \\
(n=62)\end{array}$ & $\begin{array}{l}\text { High NLR } \\
(n=61)\end{array}$ & $p$ value \\
\hline Age, years & $54.7 \pm 11.83$ & $51.1 \pm 12.59$ & 0.1 \\
\hline \multicolumn{4}{|l|}{ Race } \\
\hline Caucasian & $5(8 \%)$ & $12(20 \%)$ & 0.2 \\
\hline Black & $22(35 \%)$ & $14(23 \%)$ & 0.2 \\
\hline Hispanic & $35(56 \%)$ & $35(57 \%)$ & 1 \\
\hline Smoking history & $14(23 \%)$ & $16(26 \%)$ & 0.8 \\
\hline \multicolumn{4}{|l|}{ cT stage } \\
\hline T0-1 & $23(37 \%)$ & $19(31 \%)$ & 0.7 \\
\hline $\mathrm{T} 2$ & $26(42 \%)$ & $19(31 \%)$ & 0.3 \\
\hline T3-4 & $13(21 \%)$ & $23(38 \%)$ & 0.048 \\
\hline \multicolumn{4}{|l|}{$\mathrm{cN}$ stage } \\
\hline No & $41(66 \%)$ & $34(56 \%)$ & 0.3 \\
\hline N1 & $13(21 \%)$ & $19(31 \%)$ & 0.2 \\
\hline N2-3 & $7(11 \%)$ & $9(15 \%)$ & 0.6 \\
\hline \multicolumn{4}{|l|}{ pT stage } \\
\hline T0 & $5(8 \%)$ & $11(18 \%)$ & 0.1 \\
\hline $\mathrm{T} 1$ & $25(40 \%)$ & $23(38 \%)$ & 0.9 \\
\hline $\mathrm{T} 2$ & $24(39 \%)$ & $17(28 \%)$ & 0.3 \\
\hline T3-4 & $8(13 \%)$ & $10(16 \%)$ & 0.6 \\
\hline \multicolumn{4}{|l|}{$\mathrm{pN}$ stage } \\
\hline $\mathrm{pNO}$ & $39(63 \%)$ & $38(62 \%)$ & 1 \\
\hline $\mathrm{pN} 1$ & $13(21 \%)$ & $13(21 \%)$ & 1 \\
\hline pN2-3 & $10(16 \%)$ & $9(15 \%)$ & 1 \\
\hline \multicolumn{4}{|l|}{ AJCC stage } \\
\hline Stage 0 & $5(8 \%)$ & $11(18 \%)$ & 0.1 \\
\hline Stage 1 & $18(29 \%)$ & $17(28 \%)$ & 1 \\
\hline Stage 2 & $27(44 \%)$ & $21(34 \%)$ & 0.3 \\
\hline Stage 3 & $12(19 \%)$ & $11(18 \%)$ & 1 \\
\hline Tumor grade (high) & $49(79 \%)$ & $47(77 \%)$ & 1 \\
\hline Total mastectomy & $27(44 \%)$ & $35(57 \%)$ & 0.2 \\
\hline Radiotherapy & $39(63 \%)$ & $39(64 \%)$ & 1 \\
\hline Chemotherapy & $50(81 \%)$ & $56(92 \%)$ & 0.8 \\
\hline Neoadjuvant chemotherapy & $22(35 \%)$ & $38(62 \%)$ & 0.003 \\
\hline Adjuvant chemotherapy & $28(45 \%)$ & $18(30 \%)$ & 0.4 \\
\hline \multicolumn{4}{|l|}{ Pathological complete } \\
\hline response & $4(18 \%)$ & $11(28 \%)$ & 0.5 \\
\hline Total leukocyte count & $6.7 \pm 1.07$ & $7.4 \pm 1.89$ & 0.01 \\
\hline Neutrophil count & $3.6 \pm 1.02$ & $5.0 \pm 1.50$ & 0.001 \\
\hline Lymphocyte count & $2.5 \pm 0.65$ & $1.8 \pm 0.57$ & 0.001 \\
\hline
\end{tabular}

tive if there were $>1 \%$ positive tumor nuclei in the sample. HER-2 status was tested by immunohistochemistry (IHC) using a semiquantitative score $(0-3+)$. Tumors with $3+$ HER2 on IHC staining were considered to show HER2 overexpression; tumors with $2+$ HER2 were further analyzed by fluorescence in situ hybridization; and those with HER2/CER17 C 2.0 were also considered to exhibit HER2 overexpression.

NLR was defined as the ratio of the absolute neutrophil count/ absolute lymphocyte count. NLR was used as continuous variable as well as categorical variable. The patients were categorized as low $(<2)$ and high NLR $(\geq 2)$, as it was found to be the median of our patients NLR values and based on the previously suggested NLR cutoff point [21]. The patients were divided into two $\mathrm{pN}$ stage groups (group 1 included patients with pN0, group 2 included $\mathrm{pN}+$ patients). Categorization of TNBC patients by NLR and $\mathrm{pN}$ status resulted in four groups of patients (group $1=$ low NLR/pN0, $2=$ high NLR/pN0, 3 = low NLR/pN+, and $4=$ high NLR/pN+).
Azab et al. 
The 5-Year Overall Survival in Triple Negative Breast Cancer Patients.

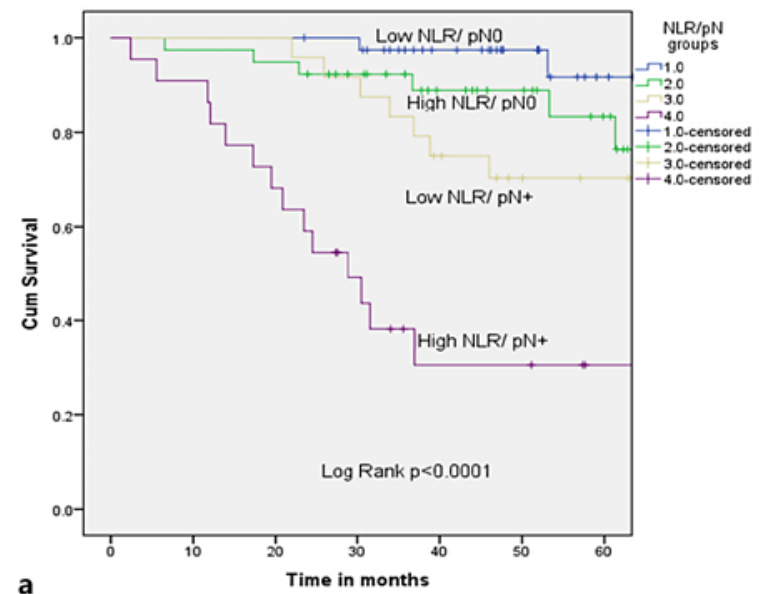

a

The 5.Year Overall Survival in Triple Negative Breast Cancer Patients with Low

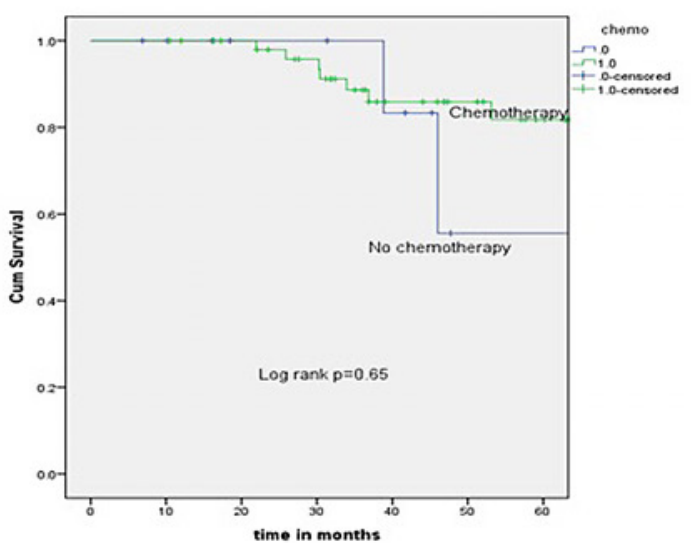

The 5-Year Overall Survival in Triple Negative Breast Cancer Patients with High Pretreatment NLR.

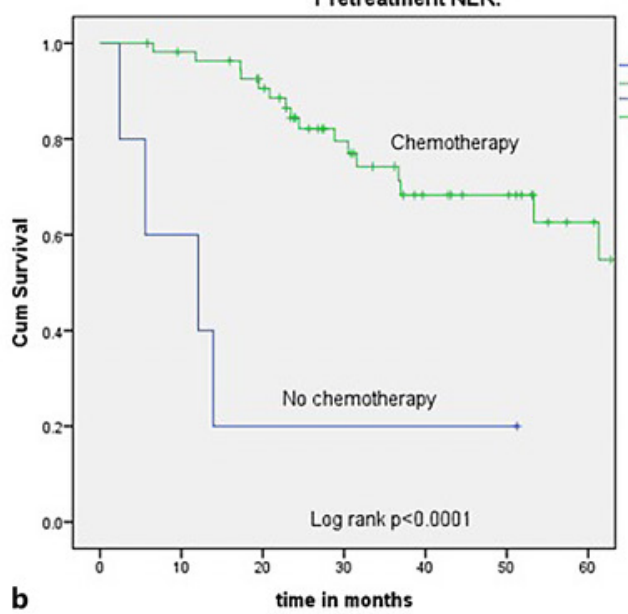

The 5-Year Disease-Free Survival in Triple Negative Breast Cancer Patients.

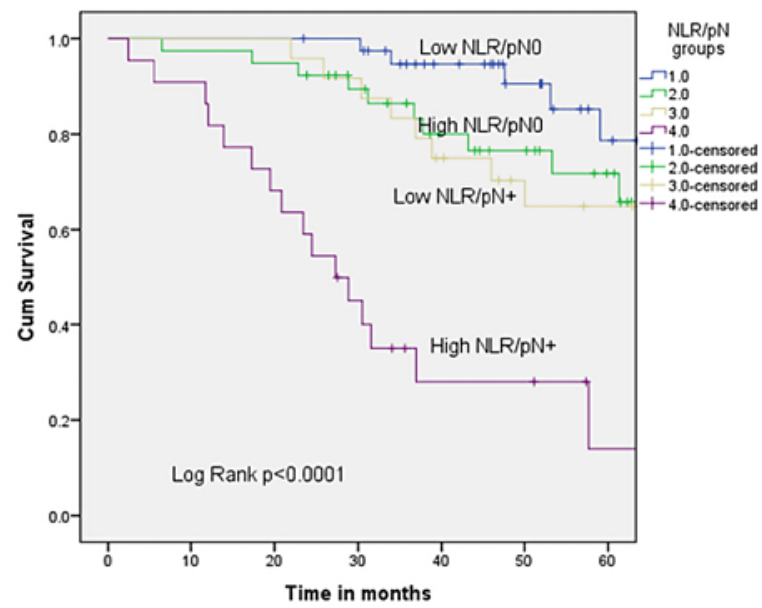

The 5-Year Disease-Free Survival in Triple Negative Breast Cancer Patients with

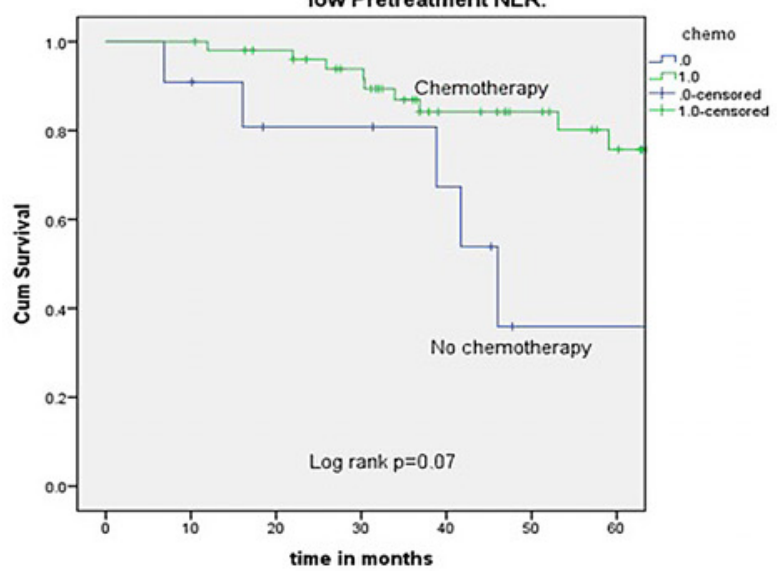

The 5-Year Disease-Free Survival in Triple Negative Breast Cancer Patients with High Pretreatment NLR.

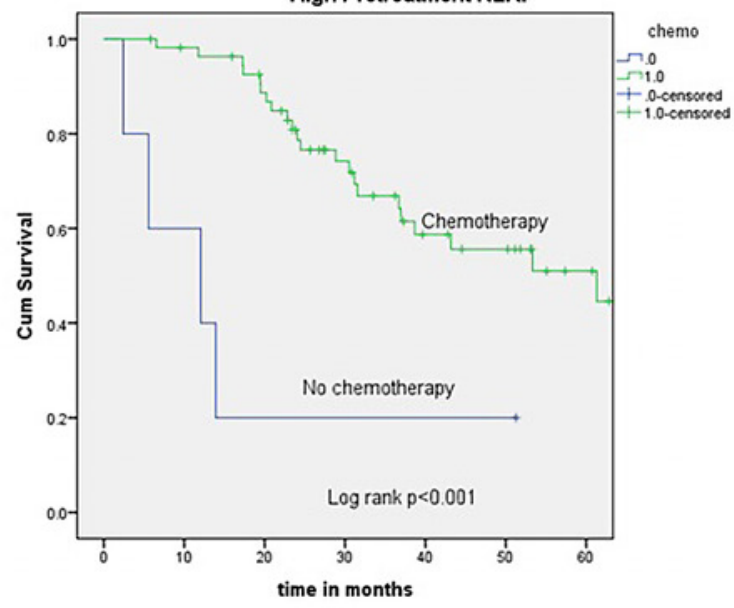

Fig. 1. a The 5-year OS and DFS statuses according to the pretreatment NLR among pN0 TNBC patients. b The 5-year OS and DFS statuses among TNBC patients according to their chemotherapy statuses in low and high NLR subgroups. 
Table 2. Cox regression multivariate model of the 5-year OS and DFS among 125 TNBC patients

\begin{tabular}{|c|c|c|c|c|c|c|c|c|}
\hline \multirow[b]{3}{*}{ High NLR vs. low NLR } & \multicolumn{4}{|l|}{ OS } & \multicolumn{4}{|l|}{ DFS } \\
\hline & \multirow{2}{*}{$\begin{array}{l}\mathrm{HR} \\
5.53\end{array}$} & \multicolumn{2}{|c|}{$95 \% \mathrm{CI}$} & \multirow{2}{*}{$\begin{array}{l}p \text { value } \\
0.0001\end{array}$} & \multirow{2}{*}{$\begin{array}{l}\mathrm{HR} \\
5.21\end{array}$} & \multicolumn{2}{|c|}{$95 \% \mathrm{CI}$} & \multirow{2}{*}{$\frac{p \text { value }}{0.0001}$} \\
\hline & & 2.23 & 13.71 & & & 2.35 & 11.56 & \\
\hline Age $>60$ years & 2.09 & 0.83 & 5.23 & 0.12 & 1.65 & 0.79 & 3.45 & 0.18 \\
\hline pT $1-2$ vs. pT $3-4$ & 0.51 & 0.25 & 1.08 & 0.08 & 0.37 & 0.20 & 0.69 & 0.002 \\
\hline pN0 vs. pN+ & 8.09 & 3.46 & 18.92 & 0.0001 & 4.60 & 2.36 & 8.95 & 0.0001 \\
\hline Chemotherapy & 0.25 & 0.08 & 0.78 & 0.02 & 0.26 & 0.10 & 0.67 & 0.005 \\
\hline Radiotherapy & 0.82 & 0.39 & 1.73 & 0.60 & 0.84 & 0.44 & 1.58 & 0.58 \\
\hline
\end{tabular}

HR, hazard ratio; CI, confidence interval; NLR, neutrophil/lymphocyte ratio; $\mathrm{pT}$, pathological T stage; $\mathrm{pN}$, negative pathological lymph node; $\mathrm{pN}+$, positive pathological lymph node.

Table 1 shows the patient characteristics according to the two NLR groups. Continuous variables are presented as mean \pm standard error, and categorical variables are presented as frequencies and percentages. For group comparisons, $\chi^{2}$ and Fisher's exact tests were used for categorical variables, and ANOVA and Kruskal-Wallis tests were used for continuous variables (depending upon the distribution of the continuous variable). Statistical analysis was performed using SPSS (version 16.0, SPSS, Inc., Chicago, IL, USA). Kaplan-Meier survival curves were plotted to illustrate difference in the OS and DFS. Statistical difference in the survival curves was evaluated using the log rank test. The Cox regression hazards model was used to build a multivariate model in order to evaluate the independent effect of NLR on mortality. All probabilities are two-sided, and $p$ values $<0.05$ were considered statistically significant.

\section{Results}

A total of 123 women with TNBC fulfilled the inclusion criteria. The average follow-up time was 54 months (range 24-110). There were 62 patients with low NLR $(<2)$ and 61 patients with high NLR $(\geq 2)$. Both low and high NLR groups had no statistically significant difference in most of their clinicopathological and treatment variables (Table 1). However, patients with high NLR had statistically significant higher rates of advanced cT stages and neoadjuvant treatment. Though the high NLR group had a higher rate of complete pathological response (11 of 38 [29\%]) compared to the low NLR group (4 of 22 [18\%]), this was not statistically significant.

The lower NLR group had better OS and DFS (53 of 62 [87\%] and 46 of 62 [74\%]) than those for the high NLR group (41/61 [67\%] and 33/61 [54\%]). In Cox regression analysis, the high NLR group had worse OS (HR 2.8, CI $1.3-5.9, p<0.001$ ) and worse DFS (HR 2.3, CI 1.2-4.2, $p<0.001)$ than the low NLR group. Moreover, categorization of TNBC patients by NLR and $\mathrm{pN}$ status resulted in four groups of patients with different Kaplan-Meier survival curves (Fig. 1a). Patients with a positive $\mathrm{pN}$ status and high NLR ( $\mathrm{pN}+$ /high NLR) had the worst survival, while those with a negative $\mathrm{pN}$ status and low NLR ( $\mathrm{pN} 0 /$ low NLR) had the best survival (log rank $p<0.0001)$.

Even after adjusting for age, $\mathrm{pT}$ and $\mathrm{pN}$ stages, radiotherapy and chemotherapy status, on Cox regression multivariate analysis, high NLR was an independent prognostic factor for both OS (HR 5.5, CI 2.2-13.7, $p<$ 0.0001 ) and DFS (HR 5.2, CI 2.3-11.6, $p<0.0001$ ) (Table 2).

Additional analysis was performed to evaluate the impact of chemotherapy on different NLR groups. Interestingly, patients receiving chemotherapy showed statistically improved OS and DFS amongst the high NLR group $(p<0.001$ and $p<0.001$, respectively) compared to those who did not receive chemotherapy. On the contrary, the low NLR group had no statistically significant OS or DFS amongst those who received chemotherapy versus those who did not ( $p=0.65$ and $p=0.07$, respectively) (Fig. 1b).

\section{Discussion}

\section{Main Findings of the Study}

Our study showed that a high pretreatment NLR is an independent predictor of OS and DFS among TNBC patients. Though we reported the similar finding in general breast cancer (i.e., including all molecular breast cancer subtypes), we advocate its more vital significance amongst that specific group of breast cancer with no targeted therapy. Previous studies showed the negative impact of elevated NLR among TNBC [18, 19, 21, 22]. Interestingly, our study showed better survival in the high NLR group receiving chemotherapy versus those with high NLR who did not receive chemotherapy. In contrast, those with low NLR showed no statistical difference in OS or DFS regarding their chemotherapy status. To our knowledge, this is the first study to evaluate the value of NLR to predict OS and DFS among TNBC in conjunction with both adjuvant and neoadjuvant chemotherapy statuses (Table 3 ). 
Table 3. Previous studies investigated the value of the NLR among TNBC patients

\begin{tabular}{|c|c|c|c|c|c|c|c|c|}
\hline Authors & Country & $\begin{array}{l}\text { Number } \\
\text { of patients }\end{array}$ & $\begin{array}{l}\text { NLR } \\
\text { cutoff }\end{array}$ & Reported chemotherapy & $\mathrm{pCR}$ & OS & DFS & Comments \\
\hline $\begin{array}{l}\text { Asano et al. } \\
\text { [19] }\end{array}$ & Japan & 61 & 3 & neoadjuvant & $28 / 61$ & NA & NS & $\begin{array}{l}\text { They reported high pCR } \\
\text { with low NLR; also, low } \\
\text { NLR with pCR had better } \\
\text { survival than high NLR } \\
\text { with pCR ( } 2 \text { patients) }\end{array}$ \\
\hline $\begin{array}{l}\text { Bozkurt et al. } \\
\text { [22] }\end{array}$ & Turkey & 85 & 2 & adjuvant chemotherapy & NA & $\begin{array}{l}\text { HR 2.9, } \\
p=0.04\end{array}$ & $\begin{array}{l}\text { HR 5.5, } \\
p=0.01\end{array}$ & \\
\hline $\begin{array}{l}\text { Jia et al. } \\
{[21]}\end{array}$ & China & 225 & 2 & NA & NA & $\begin{array}{l}\text { HR 3.1, } \\
p=0.04\end{array}$ & $\begin{array}{l}\text { HR 2.6, } \\
p=0.01\end{array}$ & $\begin{array}{l}\text { Multivariate analysis for all } \\
\text { molecular subtypes showed } \\
\text { OS for pN+ vs. pN0 HR } 2.5 \text {, } \\
p=0.055\end{array}$ \\
\hline $\begin{array}{l}\text { Noh et al. } \\
{[23]}\end{array}$ & Korea & 81 & 2.5 & NA & NA & NS & NS & $\begin{array}{l}\text { Multivariate analysis for } \\
\text { total patients including } \\
\text { other molecular subtypes } \\
\text { showed pN+ vs. pN0 HR } \\
11, p=0.001, \mathrm{NLR}>2.5 \mathrm{HR} \\
4, p=0.003\end{array}$ \\
\hline $\begin{array}{l}\text { Pistelli et al. } \\
\text { [18] }\end{array}$ & Italy & 90 & 3 & adjuvant chemotherapy & NA & $\begin{array}{l}\text { HR 6.2, } \\
p=0.01\end{array}$ & $\begin{array}{l}\text { HR 5.2, } \\
p=0.03\end{array}$ & $\begin{array}{l}\mathrm{pN} \text { and } \mathrm{pT} \text { status were not } \\
\text { significant in multivariate }\end{array}$ \\
\hline
\end{tabular}

NLR, neutrophil/lymphocyte ratio; pCR, pathological complete response; OS, overall survival; DFS, disease-free survival; NA, not available; NS, nonsignificant; $\mathrm{pN}$, pathological nodal stage; $\mathrm{pT}$, pathological T stage.

\section{Comparison of This Study with Previous Studies}

Similar to previous studies [18,21,22], we found that pretreatment NLR is a strong independent predictor of survival among TNBC patients. Similar findings were not found in smaller sample studies with 61 and 81 TNBC patients [19, 23]. Contrary to Asano et al. [19], who found a higher rate of pCR among the lower NLR group, our results showed a non-statistically significant higher rate of pCR among the high NLR group compared to the low NLR group ( 80 vs. $75 \%$ ). Of note Asano's study (61 patients) and our study (60 patients) had a relatively small number of TNBC patients who received neoadjuvant chemotherapy. We found a statistically significant association between high NLR and advanced T stage cancer, which was also found in other studies $[13,14,24]$. Interestingly, in some multivariate models of previous studies, high NLR was predicting mortality, while nodal status was not showing statistical significance $[13,18,21,22]$.

\section{The Role of Neutrophils and Lymphocytes in Breast}

\section{Cancer Outcome}

There is a growing body of evidence that supports the negative impact of tumor-associated neutrophils, neutrophilia, and lymphopenia and also the positive impact of tumor-infiltrating lymphocytes. The mechanism of cancer-induced neutrophilia is unclear. McGary et al. [25] demonstrated tumor-induced neutrophilia via metastatic tumor secretion of granulocyte-macrophage colonystimulating factor/interleukin-3 activity. Moreover, tumor-associated neutrophils were found to promote tumor growth and metastasis via the paradoxical effect of secreting a large amount of oncostatin $\mathrm{M}$ when neutrophil cocultured with breast cancer cells [9]. Brandau et al. [26] described the two sides of the neutrophil effect on tumor (protumor and antitumor functions). Interestingly, Coffelt et al. [27] suggest an absence of this two sides view. Furthermore, they proposed several neutrophil protumor mechanisms, including cancer initiation and progression, and their potential as clinical surrogate and therapeutic targets. On the contrary, tumor-infiltrating lymphocytes are associated with better survival particularly among TNBC patients [28]. Ohtani et al. [29] described the favorable outcome and better survival associated with lymphocyte-predominant (lymphocyte/stroma ratio) breast cancer, especially among TNBC. Additionally, lymphopenia was associated with worse OS among various types of cancer, including breast cancer [30]. Our higher NLR group had a higher rate of advanced $\mathrm{T}$ stages that corroborates the aforementioned reciprocal relationship between tumor burden and both peripheral neutrophil and lymphocyte counts. Of note, the higher rate of advanced $\mathrm{T}$ stages among the higher NLR group explains the higher rate of neoadjuvant chemotherapy in the same group compared to lower NLR patients. 


\section{Utilization of NLR in TNBC Patients}

In our study, we have found two potential utilizations of NLR. First, categorization of patients into four groups based on their NLR and $\mathrm{pN}$ statuses provides better risk stratification for TNBC patients. We advocate the combining of NLR and $\mathrm{pN}$ in a risk stratification model representing the tumor and host factors. Second, utilization potential was our finding of a better OS and DFS with chemotherapy among the high NLR group only. These findings need further research to validate and possible direct the treatment modalities. Over the last decade, several studies have demonstrated the positive impact of normalizing NLR after chemotherapy on the survivals of cancer patients [31-33]. Contrary to prior studies [19, 34], we did not observe a difference in PCR in both NLR groups but rather a non-statistically significant higher rate of pCR among high NLR.

\section{Cuttoff Points and Use of NLR as Continuous \\ Variables}

Of note, our cutoff point was lower than in previous papers $[13,14,18]$, as our population included a higher rate of Hispanics and less Caucasians. Our previous paper on a US representative population sample, demonstrated that both Hispanics and Blacks had a lower mean NLR (1.8 and 2.1, respectively) compared to the mean NLR (2.2) of non-Hispanic Whites [35]. We advocate that NLR should be used with a careful consideration to these racial differences.

\section{Limitations}

Though our study included more TNBC patients than previous NLR/TNBC papers, we acknowledge that the total number is relatively small. The retrospective nature of the study precludes us from proper studying other variables (i.e., NLR change after chemotherapy). Despite adjusting for the confounding variables in multivariate analyses models, the higher rate of advanced $\mathrm{T}$ stages and neoadjuvant chemotherapy among higher NLR groups could be responsible for potential biases in the final results. Our study included less Caucasians.

\section{Conclusion}

Our study showed that high pretreatment NLR is an independent predictor of poor OS and DFS amongst TNBC patients. Furthermore, combining NLR and pN provides better risk stratification for TNBC patients than each factor alone. Chemotherapy showed survival benefits only in patients with high NLR but not in patients with low NLR. Our findings suggest a possible integration of NLR in the treatment plan for TNBC patients. Larger prospective studies are needed to validate these findings.

\section{Acknowledgement}

We like to acknowledge the sincere efforts of the tumor registry and Mr. Herna Stuart for their dedication to support research and maintain high quality cancer registry baseline data.

\section{Statement of Ethics}

The authors obtained study protocol approval from our institute's committee for the above research. Informed consent was omitted given the retrospective nature of the conducted research.

\section{Disclosure Statement}

There is no relationship with any industry to disclose. The authors report no potential conflicts of interest.

\section{Funding Sources}

The authors received no funding for this study.

\section{Author Contributions}

All authors have read the manuscript and approved its final submission. Dr. Azab, Dr. Amundson, Dr. Cioci, Dr. Stuart, and Dr. Franceschi contributed to the conception, analysis, and drafting of the manuscript. Dr. Yakoub, Dr. Moffat, Dr. Avisar, Dr. Livingstone, and Dr. Franceschi reviewed and re-edited the manuscript.

\section{References}

1 Siegel RL, Miller KD, Jemal A. Cancer Statistics, 2017. CA Cancer J Clin. 2017 Jan;67(1): 7-30.

2 Bauer KR, Brown M, Cress RD, Parise CA Caggiano V. Descriptive analysis of estrogen receptor (ER)-negative, progesterone receptor (PR)-negative, and HER2-negative invasive breast cancer, the so-called triple-negative phenotype: a population-based study from the California cancer Registry. Cancer. 2007 May;109(9):1721-8.
3 Foulkes WD, Smith IE, Reis-Filho JS. Triplenegative breast cancer. N Engl J Med. 2010 Nov;363(20):1938-48.

4 Lehmann BD, Bauer JA, Chen X, Sanders ME, Chakravarthy AB, Shyr Y, et al. Identification of human triple-negative breast cancer subtypes and preclinical models for selection of targeted therapies. J Clin Invest. 2011 Jul; 121(7):2750-67.
5 Masuda H, Baggerly KH, Wang Y, Zhang Y, Gonzalez-Angulo AM, Meric-Bernstam F, Valero V, Lehmann BD, Pietenpol JA, Hortobagy GN, Fraser Symmans W, Ueno NT. Differential Response to Neoadjuvant Chemotherapy Among 7 Triple-Negative Breast Cancer Molecular Subtypes. Clin Cancer Res 2013 Oct;19(19):5533-40.

6 Carey LA, Dees EC, Sawyer L, Gatti L, Moore DT, Collichio F, et al. The triple negative paradox: primary tumor chemosensitivity of breast cancer subtypes. Clin Cancer Res. 2007 Apr;13(8):2329-34. 
7 Balkwill F, Mantovani A. Inflammation and cancer: back to Virchow? Lancet. 2001 Feb; 357(9255):539-45.

8 Mantovani A, Marchesi F, Porta C, Sica A, Allavena P. Inflammation and cancer: breast cancer as a prototype. Breast. 2007 Dec;16 Suppl 2:S27-33.

9 Queen MM, Ryan RE, Holzer RG, Keller-Peck CR, Jorcyk CL. Breast cancer cells stimulate neutrophils to produce oncostatin M: potential implications for tumor progression. Cancer Res. 2005 Oct;65(19):8896-904.

10 Pierce BL, Ballard-Barbash R, Bernstein L, Baumgartner RN, Neuhouser ML, Wener $\mathrm{MH}$, et al. Elevated biomarkers of inflammation are associated with reduced survival among breast cancer patients. J Clin Oncol. 2009 Jul;27(21):3437-44.

11 Bachelot T, Ray-Coquard I, Menetrier-Caux C, Rastkha M, Duc A, Blay JY. Prognostic value of serum levels of interleukin 6 and of serum and plasma levels of vascular endothelial growth factor in hormone-refractory metastatic breast cancer patients. Br J Cancer. 2003 Jun;88(11):1721-6.

12 Al Murri AM, Bartlett JM, Canney PA, Doughty JC, Wilson C, McMillan DC. Evaluation of an inflammation-based prognostic score (GPS) in patients with metastatic breast cancer. Br J Cancer. 2006 Jan;94(2):227-30.

13 Azab B, Bhatt VR, Phookan J, Murukutla S, Kohn N, Terjanian T, et al. Usefulness of the neutrophil-to-lymphocyte ratio in predicting short- and long-term mortality in breast cancer patients. Ann Surg Oncol. 2012 Jan;19(1): 217-24.

14 Azab B, Shah N, Radbel J, Tan P, Bhatt V, Vonfrolio S, et al. Pretreatment neutrophil/ lymphocyte ratio is superior to platelet/lymphocyte ratio as a predictor of long-term mortality in breast cancer patients. Med Oncol. 2013 Mar;30(1):432-4.

15 Wei B, Yao M, Xing C, Wang W, Yao J, Hong $\mathrm{Y}$, et al. The neutrophil lymphocyte ratio is associated with breast cancer prognosis: an updated systematic review and meta-analysis. OncoTargets Ther. 2016 Sep;9:5567-75.

16 Orditura M, Galizia G, Diana A, Saccone C, Cobellis L, Ventriglia J, et al. Neutrophil to lymphocyte ratio (NLR) for prediction of distant metastasis-free survival (DMFS) in early breast cancer: a propensity score-matched analysis.ESMOOpen.2016Mar;1(2):e000038.

17 Ethier JL, Desautels D, Templeton A, Shah PS, Amir E. Prognostic role of neutrophil-tolymphocyte ratio in breast cancer: a systematic review and meta-analysis. Breast Cancer Res. 2017 Jan;19(1):2.
18 Pistelli M, De Lisa M, Ballatore Z, Caramanti M, Pagliacci A, Battelli N, et al. Pre-treatment neutrophil to lymphocyte ratio may be a useful tool in predicting survival in early triple negative breast cancer patients. BMC Cancer. 2015 Mar;15(1):195.

19 Asano Y, Kashiwagi S, Onoda N, Noda S, Kawajiri H, Takashima T, et al. Predictive Value of Neutrophil/Lymphocyte Ratio for Efficacy of Preoperative Chemotherapy in Triple-Negative Breast Cancer. Ann Surg Oncol. 2016 Apr;23(4):1104-10.

20 Green MC, Buzdar AU, Smith T, Ibrahim NK, Valero V, Rosales MF, et al. Weekly paclitaxel improves pathologic complete remission in operable breast cancer when compared with paclitaxel once every 3 weeks. J Clin Oncol. 2005 Sep;23(25):5983-92.

21 Jia W, Wu J, Jia H, Yang Y, Zhang X, Chen K, et al. The peripheral blood neutrophil-tolymphocyte ratio is superior to the lymphocyte-to-monocyte ratio for predicting the long-term survival of triple-negative breast cancer patients. PLoS One. 2015 Nov; 10(11):e0143061.

22 Bozkurt O, Karaca H, Berk V, Inanc M, Ocak Duran A, Ozaslan E, et al. Predicting the role of the pretreatment neutrophil to lymphocyte ratio in the survival of early triple-negative breast cancer patients. J BUON. 2015 NovDec;20(6):1432-9.

23 Noh H, Eomm M, Han A. Usefulness of pretreatment neutrophil to lymphocyte ratio in predicting disease-specific survival in breast cancer patients. J Breast Cancer. 2013 Mar; 16(1):55-9.

24 Ulas A, Avci N, Kos T, Cubukcu E, Olmez OF, Bulut N, et al. Are neutrophil/lymphocyte ratio and platelet/lymphocyte ratio associated with prognosis in patients with HER2-positive early breast cancer receiving adjuvant trastuzumab? J BUON. 2015 May-Jun;20(3): 714-22.

25 McGary CT, Miele ME, Welch DR. Highly metastatic $13762 \mathrm{NF}$ rat mammary adenocarcinoma cell clones stimulate bone marrow by secretion of granulocyte-macrophage colonystimulating factor/interleukin-3 activity. Am J Pathol. 1995 Dec;147(6):1668-81.

26 Brandau S, Dumitru CA, Lang S. Protumor and antitumor functions of neutrophil granulocytes. Semin Immunopathol. 2013 Mar; 35(2):163-76
27 Coffelt SB, Wellenstein MD, de Visser KE. Neutrophils in cancer: neutral no more. Nat Rev Cancer. 2016 Jul;16(7):431-46.

28 Dushyanthen S, Beavis PA, Savas P, Teo ZL Zhou C, Mansour M, et al. Relevance of tumor-infiltrating lymphocytes in breast cancer. BMC Med. 2015 Aug;13(1):202.

29 Ohtani H, Mori-Shiraishi K, Nakajima M, Ueki H. Defining lymphocyte-predominant breast cancer by the proportion of lymphocyte-rich stroma and its significance in routine histopathological diagnosis. Pathol Int. 2015 Dec;65(12):644-51.

30 Ray-Coquard I, Cropet C, Van Glabbeke M, Sebban C, Le Cesne A, Judson I, Tredan O, Verweij J, Biron P, Labidi I, Guastalla JP, Bachelot T, Perol D, Chabaud S, Hogendoorn PC, Cassier P, Dufresne A, Blay JY; European Organization for Research and Treatment of Cancer Soft Tissue and Bone Sarcoma Group. Lymphopenia as a prognostic factor for overall survival in advanced carcinomas, sarcomas, and lymphomas. Cancer Res. 2009 Jul; 69(13):5383-91.

31 Chua W, Charles KA, Baracos VE, Clarke SJ. Neutrophil/lymphocyte ratio predicts chemotherapy outcomes in patients with advanced colorectal cancer. Br J Cancer. 2011 Apr;104(8):1288-95.

32 Wu Y, Li C, Zhao J, Yang L, Liu F, Zheng H, et al. Neutrophil-to-lymphocyte and plateletto-lymphocyte ratios predict chemotherapy outcomes and prognosis in patients with colorectal cancer and synchronous liver metastasis. World J Surg Oncol. 2016 Nov; 14(1): 289

33 Kishi Y, Kopetz S, Chun YS, Palavecino M, Abdalla EK, Vauthey JN. Blood neutrophilto-lymphocyte ratio predicts survival in patients with colorectal liver metastases treated with systemic chemotherapy. Ann Surg Oncol. 2009 Mar;16(3):614-22.

34 Chen Y, Chen K, Xiao X, Nie Y, Qu S, Gong $\mathrm{C}$, et al. Pretreatment neutrophil-to-lymphocyte ratio is correlated with response to neoadjuvant chemotherapy as an independent prognostic indicator in breast cancer patients: a retrospective study. BMC Cancer. 2016 May;16(1):320.

35 Azab B, Camacho-Rivera M, Taioli E. Average values and racial differences of neutrophil lymphocyte ratio among a nationally representative sample of United States subjects. PLoS One. 2014 Nov;9(11):e112361. 